\title{
POSITIVELY CURVED COMPLEX SUBMANIFOLDS IMMERSED IN A COMPLEX SPACE FORM
}

\author{
TAKEHIRO ITOH
}

Dedicated to Professor T. Otsuki on his 60th birthday

\begin{abstract}
The author gives the partial solution for the conjecture; a Kaehler submanifold in a complex space form of constant holomorphic sectional curvature 1 is totally geodesic if ever its holomorphic sectional curvature is greater than $\frac{1}{2}$.
\end{abstract}

Let $\tilde{M}_{m}(\tilde{c})$ be an $m$-dimensional complex space form of constant holomorphic sectional curvature $\tilde{c}$. Let $M_{n}$ be an $n$-dimensional Kaehler submanifold of $\tilde{M}_{m}(\tilde{c})$. Then we have had the following conjecture:

If all holomorphic sectional curvatures of $M_{n}$ are greater than $\tilde{c} / 2$, then $M_{n}$ is totally geodesic.

In [3], K. Ogiue gave some partial results for this conjecture.

In this paper, we shall prove the following partial solution for the above conjecture,

THEOREM. Let $M$ be an $n$-dimensional complete Kaehler submanifold immersed in an m-dimensional complex space form of constant holomorphic sectional curvature $\tilde{c}$. If every holomorphic sectional curvature of $M$ (with respect to the induced metric) is greater than $3 n \tilde{c} /(4 n+2)$, then $M$ is totally geodesic.

1. Preliminaries. Let $\tilde{M}$ be an $(n+p)$-dimensional complex space form of constant holomorphic sectional curvature $\tilde{c}$ and $M$ be an $n$-dimensional Kaehler submanifold immersed in $\tilde{M}$ (i.e., complex submanifold with the induced Kaehler structure). Let $J$ (resp. $\tilde{J}$ ) be the complex structure of $M$ (resp. $\tilde{M})$ and $g$ (resp. $\tilde{g})$ be the Kaehler metric of $M$ (resp. $\tilde{M}$ ). We denote by $\nabla$ (resp. $\tilde{\nabla}$ ) the covariant differentiation with respect to $g$ (resp. $\tilde{g}$ ). Then the second fundamental form $\sigma$ of the immersion is given by

$$
\sigma(X, Y)=\tilde{\nabla}_{X} Y-\nabla_{X} Y \text { for vector fields } X \text { and } Y \text { on } M,
$$

and it satisfies

$$
\sigma(X, Y)=\sigma(Y, X), \quad \sigma(J X, Y)=\sigma(X, J Y)=\tilde{J}(\sigma(X, Y)) .
$$

We choose a local field of orthonormal frames $e_{1}, e_{2}, \ldots, e_{n}, e_{1^{*}}=$ $J e_{1}, \ldots, e_{n^{*}}=J e_{n}, e_{1}, e_{\tilde{2}}, \ldots, e_{\tilde{p}}, e_{1^{*}}=\tilde{J} e_{1}, \ldots, e_{\tilde{p}^{*}}=\tilde{J} e_{\tilde{p}}$ in $\tilde{M}$ in such a way that, restricted to $M, e_{1}, \ldots, e_{n^{*}}$ are tangent to $M$. With respect to the

Received by the editors November 28, 1977.

AMS (MOS) subject classifications (1970). Primary 53C55, 53C40.

Key words and phrases. Kaehler submanifold, Holomorphic pinching.

ㅅ. American Mathematical Society 1978 
frame fields chosen above, let $\omega_{1}, \ldots, \omega_{n}, \omega_{1^{*}}, \ldots, \omega_{n^{*}}$, $\omega_{\tilde{1}}, \ldots, \omega_{\tilde{p}}, \omega_{1^{*}}, \ldots, \omega_{\tilde{p}^{*}}$ be the field of dual frames. Then, restricting these forms to $M$, we have the structure equations of $M:^{1}$

$$
\left\{\begin{array}{l}
\omega_{\lambda}=0, \quad \omega_{i \lambda}=\sum h_{i j}^{\lambda} \omega_{j}, \quad h_{i j}^{\lambda}=h_{j i}^{\lambda}, \\
d \omega_{i}=\sum \omega_{i j} \wedge \omega_{j}, \quad \omega_{i j}+\omega_{j i}=0 \\
d \omega_{i j}=\sum \omega_{i k} \wedge \omega_{k j}-\Omega_{i j}, \quad \Omega_{i j}=\frac{1}{2} \sum R_{i j k l} \omega_{k} \wedge \omega_{l} .
\end{array}\right.
$$

The equation of Gauss is written as

$$
\begin{aligned}
R_{i j k l}= & \frac{\tilde{c}}{4}\left(\delta_{i k} \delta_{j l}-\delta_{i l} \delta_{j k}+J_{i k} J_{j l}-J_{i l} J_{j k}+2 J_{i j} J_{k l}\right) \\
& +\sum\left(h_{i k}^{\lambda} h_{j l}^{\lambda}-h_{i l}^{\lambda} h_{j k}^{\lambda}\right),
\end{aligned}
$$

where

$$
J=\left(J_{i j}\right)=\left(\begin{array}{cc}
0 & -I_{n} \\
I_{n} & 0
\end{array}\right),
$$

$I_{n}$ being the identity matrix. Let $H_{\alpha}=\left(h_{i j}^{\alpha}\right), H_{\alpha}^{\prime}=\left(h_{a b}^{\alpha}\right)$ and $H_{\alpha}^{\prime \prime}=\left(h_{a b}^{\alpha}\right)$, then we can easily see that

$$
H_{\alpha}=\left(\begin{array}{cc}
H_{\alpha}^{\prime} & H_{\alpha}^{\prime \prime} \\
H_{\alpha}^{\prime \prime} & -H_{\alpha}^{\prime}
\end{array}\right) \text { and } H_{\alpha^{*}}=\left(\begin{array}{cc}
-H_{\alpha}^{\prime \prime} & H_{\alpha}^{\prime} \\
H_{\alpha}^{\prime} & H_{\alpha}^{\prime \prime}
\end{array}\right)
$$

Now, we can consider the following nonnegative functions on $M$;

$$
K_{N}=\sum\left(\sum_{k}\left(h_{i k}^{\lambda} h_{k j}^{\mu}-h_{j k}^{\lambda} h_{k i}^{\mu}\right)\right)^{2} \text { and } L_{N}=\sum h_{i j}^{\lambda} h_{i j}^{\mu} h_{k l}^{\lambda} h_{k l}^{\mu} .
$$

Then we know the following differential equation [1]:

$$
\frac{1}{2} \Delta\|\sigma\|^{2}=\sum\left\|\nabla^{\prime} \sigma\right\|^{2}-K_{N}-L_{N}+\frac{n+2}{2} \tilde{c}\|\sigma\|^{2},
$$

where $\|\phi\|$ is the length of a form $\phi$ and $\nabla^{\prime}$ is the covariant differentiation respect to the connection in (tangent bundle of $M$ ) $\oplus$ (normal bundle) induced naturally from $\tilde{\nabla}$.

2. Proof of Theorem. First of all we note that, by the theorem of Myers [3], $M$ is compact. Let $c$ be the minimum of all holomorphic sectional curvatures of $M$. The holomorphic sectional curvature $H(X)$ of $M$ determined by a unit vector $X$ is given by

$$
H(X)=\tilde{c}-2\|\sigma(X, X)\|^{2}
$$

\footnotetext{
'We use the following convention on the range of indices unless otherwise stated: $a, b, c=$ $1,2, \ldots, n ; i, j, k, l=1,2, \ldots, n, 1^{*}, \ldots, n^{*} ; \alpha, \beta, \gamma=\tilde{1}, \ldots, \tilde{p} ; \lambda, \mu, \nu=$ $\tilde{1}, \tilde{2}, \ldots, \tilde{p}, \tilde{1}^{*}, \tilde{2}^{*}, \ldots, \tilde{p}^{*}$; and we agree that repeated indices under a summation sign without indication are summed over the respective range.
} 
It is clear from (2.1) that $\tilde{c} \geqslant c$. Setting $A_{i j}:=\sigma\left(e_{i}, e_{j}\right)$, from (1.2) we have

$$
A_{a^{*} b^{*}}=-A_{a b}, \quad A_{a b^{*}}=A_{a^{*} b}=\tilde{J} A_{a b}, \text { for } a, b .
$$

For a vector $X=\lambda e_{i}+\eta e_{j}$ on $M$, we get

$$
\sigma(X, X)=\lambda^{2} A_{i i}+2 \lambda \eta A_{i j}+\eta^{2} A_{i j} .
$$

Since $H(X) \geqslant c$ for all unit vector $X,\|\sigma(X, X)\| \leqslant(\tilde{c}-c) / 2$, in particular,

$$
\left\|A_{a a}\right\|^{2}=\left\|\sigma\left(e_{a}, e_{a}\right)\right\|^{2} \leqslant(\tilde{c}-c) / 2 \text { for } \forall a .
$$

Since $\|\sigma(X, X)\| \leqslant(\tilde{c}-c) / 2$ for all unit vectors $X=\lambda e_{i}+\eta e_{j}(i \neq j)$, given by

\begin{tabular}{|l|l||c|c|}
\hline$i$ & $j$ & $\lambda$ & $\eta$ \\
\hline \multirow{2}{*}{$a$} & \multirow{2}{*}{$b$} & $\lambda$ & $\eta$ \\
\cline { 3 - 4 } & & $\lambda$ & $-\eta$ \\
\hline \multirow{2}{*}{$a$} & \multirow{2}{*}{$b^{*}$} & $\lambda$ & $\eta$ \\
\cline { 3 - 4 } & & $\lambda$ & $-\eta$ \\
\hline
\end{tabular}

\begin{tabular}{|l|l||r|r|}
\hline$i$ & $j$ & $\lambda$ & $\eta$ \\
\hline \multirow{2}{*}{$b$} & \multirow{2}{*}{$a$} & $\lambda$ & $\eta$ \\
\cline { 3 - 4 } & & $\lambda$ & $-\eta$ \\
\hline \multirow{2}{*}{$b$} & \multirow{2}{*}{$a^{*}$} & $\lambda$ & $\eta$ \\
\cline { 3 - 4 } & & $\lambda$ & $-\eta$ \\
\hline
\end{tabular}

we have

$$
\left(\lambda^{4}+\eta^{4}\right)\left(\left\|A_{a a}\right\|^{2}+\left\|A_{b b}\right\|^{2}\right)+8 \lambda^{2} \eta^{2}\left\|A_{a b}\right\|^{2} \leqslant \tilde{c}-c .
$$

Since $\lambda^{4}+\eta^{4} \geqslant 2 \lambda^{2} \eta^{2}$, from (2.4) we get

$$
\lambda^{2} \eta^{2}\left(\left\|A_{a a}\right\|^{2}+\left\|A_{b b}\right\|^{2}+4\left\|A_{a b}\right\|^{2}\right) \leqslant(\tilde{c}-c) / 2
$$

in which, setting $\lambda^{2}=\eta^{2}=\frac{1}{2}$, we get

$$
\left\|A_{a a}\right\|^{2}+\left\|A_{b b}\right\|^{2}+4\left\|A_{a b}\right\|^{2} \leqslant 2(\tilde{c}-c) .
$$

Since $\left\|A_{a a}\right\|^{2}+\left\|A_{b b}^{2}\right\| \geqslant 2\left\|A_{a a}\right\| \cdot\left\|A_{b b}\right\|,(2.5)$ implies

$$
\left\|A_{a a}\right\| \cdot\left\|A_{b b}\right\|+2\left\|A_{a b}\right\|^{2} \leqslant \tilde{c}-c .
$$

Using (2.3), (2.6) and the fact that $\left|\left\langle A_{a a}, A_{b b}\right\rangle\right| \leqslant\left\|A_{a a}\right\| \cdot\left\|A_{b b}\right\|$, we can prove

LEMMA. The following inequality holds on $M$;

$$
K_{N}+L_{N} \leqslant(2 n+1)(\tilde{c}-c)\|\sigma\|^{2} \text {. }
$$

Proof of Lemma. For each $\alpha$, we set

$$
\begin{aligned}
& K_{N}^{\alpha}:=\sum_{i, j, \mu}\left(\sum_{k}\left(h_{i k}^{\alpha} h_{k j}^{\mu}-h_{j k}^{\alpha} h_{k i}^{\mu}\right)\right)^{2}+\sum_{i, j, \mu}\left(\sum_{k}\left(h_{i k}^{\alpha^{*}} h_{k j}^{\mu}-h_{j k}^{\alpha^{*}} h_{k i}^{\mu}\right)\right)^{2}, \\
& L_{N}^{\alpha}:=\sum_{i, j, k, l, \mu} h_{i j}^{\alpha} h_{i j}^{\mu} h_{k l}^{\alpha} h_{k l}^{\mu}+\sum_{i, j, k, l, \mu} h_{i j}^{\alpha^{*} h_{i j}^{\mu} h_{k l}^{\alpha^{*}} h_{k l}^{\mu} .}
\end{aligned}
$$

Then $K_{N}=\Sigma_{\alpha} K_{N}^{\alpha}$ and $L_{N}=\Sigma_{\alpha} L_{N}^{\alpha}$. For fixed $\alpha$, choosing a frame $\left\{e_{1}, \ldots, e_{n}, e_{1^{*}}, \ldots, e_{n^{*}}\right\}$ such that $h_{i j}^{\alpha}=\lambda_{i}^{\alpha} \delta_{i j}$, from (1.5) we have 


$$
H_{\alpha}=\left(\begin{array}{cc}
H_{\alpha}^{\prime} & 0 \\
0 & -H_{\alpha}^{\prime}
\end{array}\right), \quad H_{\alpha^{*}}=\left(\begin{array}{cc}
0 & H_{\alpha}^{\prime} \\
H_{\alpha}^{\prime} & 0
\end{array}\right) \quad \text { and } \quad H_{\alpha}^{\prime}=\left(\begin{array}{ccc}
\lambda_{1}^{\alpha} & & 0 \\
& \ddots & \\
& 0 & \lambda_{n}^{\alpha}
\end{array}\right)
$$

Using (2.8), we have

$$
\begin{aligned}
& K_{N}^{\alpha}=8 \sum_{a \neq b}\left\{\left(\lambda_{a}^{\alpha}\right)^{2}+\left(\lambda_{b}^{\alpha}\right)^{2}\right\}\left\|A_{a b}\right\|^{2}+16 \sum_{a}\left(\lambda_{a}^{\alpha}\right)^{2}\left\|A_{a a}\right\|^{2} \\
& L_{N}^{\alpha}=8 \sum_{a \neq b} \lambda_{a}^{\alpha} \lambda_{b}^{\alpha}\left\langle A_{a a}, A_{b b}\right\rangle+8 \sum_{a}\left(\lambda_{a}^{\alpha}\right)^{2}\left\|A_{a a}\right\|^{2}
\end{aligned}
$$

and so

$$
\begin{aligned}
K_{N}^{\alpha}+L_{N}^{\alpha}= & 8 \sum_{a \neq b}\left[\left\{\left(\lambda_{a}^{\alpha}\right)^{2}+\left(\lambda_{b}^{\alpha}\right)^{2}\right\}\left\|A_{a b}\right\|^{2}+\lambda_{a}^{\alpha} \lambda_{b}^{\alpha}\left\langle A_{a a}, A_{b b}\right\rangle\right] \\
& +24 \sum_{a}\left(\lambda_{a}^{\alpha}\right)^{2}\left\|A_{a a}\right\|^{2} .
\end{aligned}
$$

Since we have the following inequalities

$$
\begin{array}{ll}
2 \lambda_{a}^{\alpha} \lambda_{b}^{\alpha}\left\langle A_{a a}, A_{b b}\right\rangle \leqslant-\left\{\left(\lambda_{a}^{\alpha}\right)^{2}+\left(\lambda_{b}^{\alpha}\right)^{2}\right\}\left\langle A_{a a}, A_{b b}\right\rangle & \text { if }\left\langle A_{a a}, A_{b b}\right\rangle<0 \\
2 \lambda_{a}^{\alpha} \lambda_{b}^{\alpha}\left\langle A_{a a}, A_{b b}\right\rangle \leqslant\left\{\left(\lambda_{a}^{\alpha}\right)^{2}+\left(\lambda_{b}^{\alpha}\right)^{2}\right\}\left\langle A_{a a}, A_{b b}\right\rangle & \text { if }\left\langle A_{a a}, A_{b b}\right\rangle \geqslant 0
\end{array}
$$

and $\left|\left\langle A_{a a}, A_{b b}\right\rangle\right| \leqslant\left\|A_{a a}\right\| \cdot\left\|A_{b b}\right\|$, we get

$$
2 \lambda_{a}^{\alpha} \lambda_{b}^{\alpha}\left\langle A_{a a}, A_{b b}\right\rangle \leqslant\left\{\left(\lambda_{a}^{\alpha}\right)^{2}+\left(\lambda_{b}^{\alpha}\right)^{2}\right\}\left\|A_{a a}\right\| \cdot\left\|A_{b b}\right\| .
$$

It follows from (2.9) and (2.10) that we have

$$
\begin{aligned}
K_{N}^{\alpha}+L_{N}^{\alpha} \leqslant & 4 \sum_{a \neq b}\left\{\left(\lambda_{a}^{\alpha}\right)^{2}+\left(\lambda_{b}^{\alpha}\right)^{2}\right\}\left\{2\left\|A_{a b}\right\|^{2}+\left\|A_{a a}\right\| \cdot\left\|A_{b b}\right\|\right\} \\
& +24 \sum_{a}\left(\lambda_{a}^{\alpha}\right)^{2}\left\|A_{a a}\right\|^{2}
\end{aligned}
$$

Hence, using (2.3) and (2.6), from (2.11) we obtain

$$
\begin{aligned}
K_{N}^{\alpha}+L_{N}^{\alpha} & \leqslant 4(\tilde{c}-c)\left[\sum_{a \neq b}\left\{\left(\lambda_{a}^{\alpha}\right)^{2}+\left(\lambda_{b}^{\alpha}\right)^{2}\right\}+3 \sum_{a}\left(\lambda_{a}^{\alpha}\right)^{2}\right] \\
& =4(2 n+1)(\tilde{c}-c) \sum_{a}\left(\lambda_{a}^{\alpha}\right)^{2} \\
& =(2 n+1)(\tilde{c}-c)\left(\operatorname{Tr} H_{\alpha}^{2}+\operatorname{Tr} H_{\alpha^{*}}^{2}\right) .
\end{aligned}
$$

Since $K_{N}+L_{N}=\Sigma_{\alpha}\left(K_{N}^{\alpha}+L_{N}^{\alpha}\right)$ and $\|\sigma\|^{2}=\Sigma_{\alpha}\left(\operatorname{Tr} H_{\alpha}^{2}+\operatorname{Tr} H_{\alpha^{*}}^{2}\right),(2.12) \mathrm{im}$ plies (2.7). Q.E.D.

Proof of TheOREM. Using (2.7), from (1.6) we have

$$
\begin{aligned}
\frac{1}{2} \Delta\|\sigma\|^{2} & \geqslant\left\|\nabla^{\prime} \sigma\right\|^{2}+\frac{n+2}{2} \tilde{c}\|\sigma\|^{2}-(2 n+1)(\tilde{c}-c)\|\sigma\|^{2} \\
& \geqslant(2 n+1)\left(c-\frac{3 n \tilde{c}}{4 n+2}\right)\|\sigma\|^{2} \quad \text { on } M .
\end{aligned}
$$


Since $M$ is compact and $c>3 n \tilde{c} /(4 n+2)$, from (2.13) $\Delta\|\sigma\|^{2}=0$ on $M$. Hence we have $\|\sigma\|=0$ identically on $M$, so that $M$ is totally geodesic. Q.E.D.

\section{BIBLIOGRAPHY}

1. S. S. Chern, M. do Carmo and S. Kobayashi, Minimal submanifolds of a sphere with second fundamental form of constant length, Functional Analysis and Related Fields, Springer, New York, 1970, pp. 59-75.

2. T. Itoh, Addendum to my paper "On Veronese manifolds", J. Math. Soc. Japan 30 (1978), 73-74.

3. K. Ogiue, Differential geometry of Kaehler submanifolds, Advances in Math. 13 (1974), 73-114.

4. S. Myer, Riemannian manifolds with positive mean curvature, Duke Math. J. 8 (1941), 401-404.

Department of Mathematics, University of Tsukuba, Ibaraki 300-31, JaPAN 